\section{Web-Based Training to Improve Knowledge and Change Practice in Preventing Healthcare Infection}

To the Editor-We read with interest the development and assessment of a web-based training program to improve knowledge about central line bloodstream infections. ${ }^{1}$ The course included video demonstrations of common errors, and knowledge was assessed using follow-up questionnaires, which showed significant improvements even 3-4 months after the completion of the course. ${ }^{1}$ The authors also suggest that those who had completed the training course were significantly more likely to comply with sterile practices.

Increasingly, healthcare education is availing of the web with a blended learning approach including e-learning as well as more traditional approaches. In addition, there is increased use by healthcare professionals of handheld devices such as mobile phones, smartphones, and tablets to facilitate access to educational and professional materials. The benefits of elearning include flexibility in terms of time and place, access to multimedia-rich resources, and the potential for interactions, all of which have been incorporated into the e-learning/ blended learning program used in the National Health Service in the United Kingdom to good effect. ${ }^{2}$ We have used 2 modules of this program, adapted for medical student use, to improve the teaching of issues relating to the prevention and control of healthcare-associated infection (HCAI), with demonstrable increases in the knowledge base. ${ }^{3}$ However, the educational challenge at the postgraduate level is probably greater, but the potential impact on patient safety is more significant.

We have also developed an educational website to target deficiencies identified in audits of surgical practice relating to HCAI prevention and control. ${ }^{4}$ Together with highlighting key issues at hospital meetings and the use of posters and discussions at audit meetings, this web-based initiative has resulted in improvements in surgical practice, such as more appropriate antibiotic prophylaxis, a reduction in the number of surgical site dressings tampered with during the first 48 hours after surgery, a fall in the number of peripheral vascular catheters (PVCs) in situ for greater than 72 hours, and a greater awareness among patients about the indication for their PVCs. ${ }^{5,6}$

This is an exciting time for healthcare educationalists, given the new modalities for teaching and learning now available, in addition to the more traditional approaches such as lectures and bedside teaching. However, it is essential that any healthcare educational initiative, blended or otherwise, traditional or incorporating web-based materials, improves both knowledge and practice leading to a culture change that prioritizes the prevention of HCAI. Furthermore, there is an opportunity for the sharing of web-based materials via open access, not only in the case of our website (http://www.surginfection .com) but also through novel mHealth technologies via mobile phones, smartphones, and tablets, all resulting in the wider application and use of these contents at a global level. However, the ultimate test is to confirm that web-based material results in sustained changes in practice over an extended period of time and not just improvements in knowledge.

\section{ACKNOWLEDGMENTS}

Financial support. The work referred to was funded through a grant from the Royal College of Surgeons in Ireland through its Strategic Development Initiative.

Potential conflicts of interest. All authors report no conflicts of interest relevant to this article. All authors submitted the ICMJE Form for Disclosure of Potential Conflicts of Interest, and the conflicts that the editors consider relevant to this article are disclosed here.

\section{Hilary Humphreys, MD; ${ }^{1,2}$ Seamus McHugh, MD; ${ }^{3,4}$ Borislav D. Dimitrov, $\mathrm{PhD} ;{ }^{5}$ Seamus Cowman, $\mathrm{PhD} ;{ }^{6}$ Sean Tierney, FRCSI; ${ }^{7,8}$ Arnie D. K. Hill, $\mathbf{M C h}^{3,4}$}

Affiliations: 1. Department of Clinical Microbiology, Royal College of Surgeons in Ireland, Beaumont Hospital, Dublin, Ireland; 2. Department of Microbiology, Beaumont Hospital, Dublin, Ireland; 3. Department of Surgery, Royal College of Surgeons in Ireland, Beaumont Hospital, Dublin, Ireland; 4. Department of Surgery, Beaumont Hospital, Dublin, Ireland; 5. Department of General Practice, Royal College of Surgeons in Ireland, Dublin, Ireland; 6. Faculty of Nursing and Midwifery, Royal College of Surgeons in Ireland, Dublin, Ireland; 7. Department of Surgery, Adelaide and Meath Hospitals, Incorporating the National Children's Hospital Tallaght, Dublin, Ireland; 8. Department of Surgical Informatics, Royal College of Surgeons in Ireland, Dublin, Ireland.

Address correspondence to Hilary Humphreys, MD, Department of Clinical Microbiology, RCSI Education and Research Centre, Beaumont Hospital, PO Box 9063, Dublin 9, Ireland (hhumphreys@rcsi.ie).

Infect Control Hosp Epidemiol 2012;33(6):644-645

(C) 2012 by The Society for Healthcare Epidemiology of America. All rights reserved. 0899-823X/2012/3306-0021\$15.00. DOI: 10.1086/665717

\section{REFERENCES}

1. Comer A, Harris AD, Shardell M, et al. Web-based training improves knowledge about central line bloodstream infections. Infect Control Hosp Epidemiol 2011;32:1219-1222.

2. Pratt RJ, O'Malley B. Supporting evidence-based infection prevention and control practices in the National Health Service in England: the NHS/TVU/Intuition approach. I Hosp Infect 2007; 65(suppl 2):142-147.

3. O'Neill E, Stephens NT, Clarke E, Cox P, O'Malley B, Humphreys $H$. Use of e-learning to enhance medical students understanding and knowledge of healthcare-associated infection prevention and control. J Hosp Infect 2011;79:368-370.

4. McHugh SM, Corrigan M, Dimitrov B, et al. A targeted e-learning 
programme for surgical trainees to enhance patient safety in preventing surgical infection. J Contin Educ Health Prof 2010;30: 257-259.

5. McHugh SM, Corrigan MA, Dimitrov BD, et al. Preventing infection in general surgery: improvements through education of surgeons by surgeons. $J$ Hosp Infect 2011;78:312-316.

6. McHugh SM, Corrigan MA, Dimitrov BD, et al. Role of patient awareness prevention of peripheral vascular catheter-related bloodstream infection. Infect Control Hosp Epidemiol 2011;32: 95-96.

\section{Reply to Weber and Rutala}

To the Editor-We read with interest the commentary by Weber and Rutala. ${ }^{1}$ The authors discuss the contribution of contaminated environmental surfaces within hospitals to transmitting clinically relevant microorganisms frequently associated with healthcare infections. We welcome commentaries of this nature, since the potential for contaminated environmental surfaces to contribute to the chain of healthcare-associated infections is often overlooked and underresearched in favor of more well-publicized and documented mechanisms, primarily the contaminated hands of healthcare workers.

Self-disinfecting surfaces have been the focus of several well-conducted research studies, including studies within our research group. ${ }^{2}$ In the recent study by Karpanen et al, ${ }^{2}$ several frequently touched items-including door handles, grab rails, and toilet seats-in a hospital ward were replaced with equivalent items produced from copper alloy and sampled for microorganisms. The study demonstrated a significant reduction in the microbial bioload on copper as compared with noncopper equivalents.

As Weber and Rutala ${ }^{1}$ state in their commentary, there are limitations to the introduction of copper items in healthcare facilities; however, this is often the case for implementing many novel strategies in infection control, and one should also consider the potential benefits. The data from the study of Karpanen et $\mathrm{al}^{2}$ clearly highlight the value of self-disinfecting surfaces in reducing microbial bioloads from clinical surfaces, and proactive infection control teams may wish to give consideration to where copper surfaces may be of benefit in their respective institutions, for example, in intensive care units or areas with high numbers of immunocompromised patients. An obvious issue is the cost of purchasing and implementing copper items. To our knowledge, cost-effectiveness studies have to date not been undertaken, and investigations to address the added value of copper are therefore clearly warranted, particularly in view of the considerable costs associated with healthcare infections. ${ }^{3}$ The alternative option is perhaps the inertia approach, allowing current environmental surfaces (eg, stainless steel, plastic) to remain dominant in clinical settings. However, these surfaces do not possess any antimicrobial activity and do not contribute to breaking the chain of infection; this is not desirable, since we aspire to design out infection in the modern world.

Copper is a highly efficacious antimicrobial and, as Weber and Rutala ${ }^{1}$ state, has been effective in eliminating important healthcare-associated pathogens. In the United Kingdom, Clostridium difficile is currently one of the leading causes of healthcare-associated infections, and while the number of cases has declined over recent years, it is still a significant cause of morbidity and mortality. Weber and Rutala ${ }^{1}$ comment that copper has not been shown in vitro to kill dormant spores of $C$. difficile. However, in the study by Weaver et al, ${ }^{4}$ copper was shown to kill dormant spores of $C$. difficile within 24-48 hours. Research undertaken by our group has also shown that copper surfaces are highly effective in reducing the viable bioload of $C$. difficile vegetative cells (NCTC 11204 and ribotype 027 ) by approximately 6 logs in both clean and soiled conditions within 30 minutes at room temperature, thus limiting the time for $C$. difficile to develop hardy, resistant environmental spores. ${ }^{5,6}$ Furthermore, studies have also shown that germinating spores of bacteria are more susceptible to killing by disinfectants than dormant spores. At Aston University, we have developed a patented germination solution for $C$. difficile that renders spores susceptible to killing by common biocides, including copper. In our research, we have demonstrated that dormant spores become metabolically active and susceptible to copper surfaces in a short time period following exposure to a $C$. difficile-specific, nontoxic germination solution. Indeed, $99 \%-99.9 \%$ of germinantexposed spores (NCTC 11204 and ribotype 027) are eliminated on both clean and soiled copper surfaces within 3 hours.

Weber and Rutala ${ }^{l}$ are correct when they state that it is impractical or impossible to coat all environmental surfaces and medical devices that could be potentially contaminated with copper. However, a pragmatic approach may be to consider designated areas that may benefit from a background level of antimicrobial protection from copper surfaces supplemented with routine infection control procedures and perhaps novel germination solutions, as alluded to previously. A previous criticism of copper items has been the aesthetic issue, which may be potentially off-putting for patients, visitors, and staff. However, there are now in excess of 300 different antimicrobial copper alloys that span a wide range of colors and surface textures; these may be implemented into clinical settings without disrupting the overall appearance of the environment.

While the antimicrobial activity of copper surfaces has been clearly demonstrated, it is important to appreciate that effective infection control relies on a bundle of measures-some already implemented into clinical practice and some potentially on the horizon-and no single, stand-alone approach is effective at controlling infection. We firmly be- 